Artigo / Article

\title{
Influência do imatinibe no resultado do TMO e sua eficácia no tratamento da recaída
}

\author{
The effect of prior exposure to imatinib on transplant outcomes and its effectiveness \\ for relapse treatment
}

Afonso Celso Vigorito

\section{Introdução}

O inibidor da tirosino quinase, mesilato de imatinibe (MI), tornou-se o tratamento de primeira linha para a Leucemia Mielóide Crônica (LMC). ${ }^{1} \mathrm{O}$ estudo randomizado internacional (IRIS) comparando o interferon versus o STI571, em pacientes com diagnóstico recente de LMC em fase crônica (FC), completou 6 anos. Os pacientes randomizados para o braço do MI apresentaram resposta hematológica completa (RHC) de $97 \%$, resposta citogenética maior (RCM) de $89 \%$ e resposta citogenética completa $(\mathrm{RCC})$ de $83 \%{ }^{2}$ Os resultados são piores na fase mais avançada, onde a progressão é vista em
$40 \%$ dos pacientes em fase acelerada (FA) e em $80 \%$ em crise blástica $(\mathrm{CB})$, com uma mediana de acompanhamento de 18 meses. $^{3-7}$

O transplante de células-tronco hematopoiéticas (TCTH) é um tratamento potencialmente curativo. Para os pacientes em FC com doadores aparentados HLA idêntico, a sobrevida, em três anos, pode ser maior do que $85 \%$, em instituições selecionadas. ${ }^{8}$ Resultados semelhantes foram obtidos em pacientes mais jovens, com doadores não aparentados HLA idêntico. ${ }^{9}$

Por outro lado, os resultados para a doença na FA e CB são piores em conseqüência do aumento da mortalidade

Doutor em Clínica Médica pela Faculdade de Ciências Médicas da Unicamp-SP.

Correspondência: Afonso Celso Vigorito

Universidade Estadual de Campinas - Unicamp

Unidade de TMO HC/Hemocentro - Unicamp - CX Postal: 6198

13083-970 - Campinas-SP - Brasil

Tel.: 193521 8740; Fax: 1935218600

E-mail:afonso@unicamp.br 
relacionada ao transplante (MRT) e da recidiva, que pode alcançar $50 \%$ ou mais. ${ }^{10,11}$

Uma vantagem do TCTH é a sua capacidade de produzir uma resposta molecular completa, que pode ocorrer em até $75 \%$ dos pacientes, resposta esta que está associada a um risco menor de recidiva e a uma potencial cura da LMC. Esta resposta molecular completa, entretanto, não é usual no tratamento com o MI..$^{12-14}$

O TCTH tem sido cada vez mais utilizado como tratamento de "salvamento" para aqueles pacientes com LMC que são intolerantes ao MI, que não conseguem uma resposta citogenética completa, ou recidivam. Esta mudança na estratégia do tratamento levantou alguns questionamentos. $\mathrm{O}$ primeiro seria se o uso do MI pré-transplante aumentaria a toxicidade e influenciaria os resultados do pós-transplante. O segundo seria se a resposta subótima, ou perda de resposta ao MI, teria impacto nos resultados do transplante. Finalmente, se a presença de mutações no domínio da tirosina quinase (DTK), diagnosticadas antes do transplante, estaria associada a um pior resultado. ${ }^{1}$

Como conseqüência da grande utilização do MI como primeira linha para o tratamento da $\mathrm{LMC}$, a população de pacientes que será submetida ao TCTH mudou. Muitos pacientes usaram o MI previamente ao transplante e receberão o transplante em FC se forem intolerantes ou falharem ao MI. ${ }^{1}$ Como o TCTH é potencialmente curativo e produz uma sobrevida alta nos pacientes em FC, a estratégia do tratamento com o MI até a falha é uma opção razoável, se o MI pré-transplante não tiver um impacto negativo nos resultados. ${ }^{1}$

Dois estudos pequenos, retrospectivos, que avaliaram a mortalidade relacionada ao transplante (MRT) associada ao uso do MI pré-transplante descobriram uma maior incidência de toxicidade hepática. ${ }^{15,16}$ Entretanto, outros estudos maiores que compararam os pacientes tratados previamente com MI com um grupo histórico, que não recebeu o MI, não mostraram um aumento da toxicidade hepática após o transplante. ${ }^{1,17,18}$ Do mesmo modo, não existem relatos de atraso da pega, tanto nos transplantes com condicionamento mieloablativos ou não mieloablativos, em pacientes com LMC e leucemia linfoblástica Ph positiva. ${ }^{17-21}$ Oehler et al, ${ }^{1}$ inclusive, mostraram que os pacientes que receberam MI tiveram uma pega mielóide ligeiramente mais rápida.

Com relação à incidência e gravidade da doença do enxerto contra o hospedeiro aguda (DECHa), um estudo mostrou mais DECHa no grupo do $\mathrm{MI},{ }^{15}$ enquanto outros não demonstraram aumento tanto da $\mathrm{DECHa}$, como da doença do enxerto contra o hospedeiro crônica (DECHc).,16-19 Deininger $e t a l^{18}$ e Oehler et $a l^{1}$ demonstraram uma incidência menor da DECHDc, especificamente da forma extensa, nos pacientes que receberam o MI. O mecanismo do efeito do MI na DECHc é especulativo. O MI inibe a proliferação de linfócitos $\mathrm{T}$, a ativação de linfócitos $\mathrm{T}$ mediados pelo receptor de células $\mathrm{T}(\mathrm{RCT})$ e a resposta dos linfócitos $\mathrm{T} \mathrm{CD} 8^{+}$ao
CMV e ao Epstein-Barr vírus. ${ }^{22}$ Outros relatos recentes indicam que o MI pode ser imunossupressor já que ele inibe a função e o desenvolvimento das células dendríticas, resultando em células que não respondem ao estímulo de maturação e não desencadeiam a resposta primária de linfócitos $\mathrm{T}$ ou a resposta de linfócitos $\mathrm{T}$ a antígenos. ${ }^{23} \mathrm{En}-$ tretanto, não é claro porque este efeito persistiria nos pacientes que não estão mais tomando MI e que receberam um grande número de células $\mathrm{T}$ de origem do doador. ${ }^{1}$

Os dados disponíveis atualmente em relação à sobrevida global (SG), sobrevida livre de doença (SLD), MRT e recidiva nos pacientes tratados com MI antes do transplante mostraram que, em uma pequena série de pacientes, houve uma associação negativa do MI com estes resultados..$^{15}$ Outros estudos maiores compararam os pacientes tratados com MI com um grupo histórico. Zaucha et $a l^{17}$ mostraram que não houve diferença na SG entre o grupo tratado com o MI e o grupo histórico. No estudo de Deininger et $a l,{ }^{18} \mathrm{o}$ tratamento prévio com o MI não teve influência na SG, na sobrevida livre de progressão e na MRT. Entretanto, houve uma tendência no aumento das recidivas no grupo que recebeu MI. Um estudo maior, e mais recente, que comparou retrospectivamente 145 pacientes com LMC que receberam MI por um mínimo de três meses antes do transplante, com 231 pacientes com LMC, que não receberam o MI, mostrou que não houve diferença estatística significativa da SG, SLD, recidiva e MRT, entre o grupo tratado e não tratado. ${ }^{1} \mathrm{O}$ impacto do $\mathrm{MI}$ na $\mathrm{SG}$ após o transplante não foi dependente do estágio da doença, comparado com o grupo que não usou o MI. ${ }^{1} \mathrm{O}$ efeito deletério do tempo entre o diagnóstico e o transplante também não foi aumentado no grupo que usou o MI. ${ }^{1}$ Porém, para os pacientes em FC, a resposta ao MI, previamente ao transplante, estava associada com os resultados do pós-transplante. Os pacientes que foram transplantados com resposta subótima ou com a perda da resposta ao MI tiveram um aumento significativo da mortalidade quando comparado aos pacientes em FC que atingiram Remissão Citogenética Completa (RCC) ou Remissão Citogenética Maior (RCM). ${ }^{1}$

\section{Eficácia do MI no tratamento da recaída}

A infusão de linfócitos do doador (DLI) tornou-se o tratamento de escolha para os pacientes que recidivam após um TCTH alogênico. A remissão molecular duradoura é alcançada na maioria dos pacientes que recidivam na fase crônica. ${ }^{24-27} \mathrm{~A} \mathrm{DECH} \mathrm{e} \mathrm{a} \mathrm{aplasia} \mathrm{da} \mathrm{medula} \mathrm{continuam} \mathrm{sendo}$ as duas complicações mais importantes do DLI, mas, quando se utiliza um esquema com doses escalonadas, estes problemas são amplamentes reduzidos. ${ }^{27,28} \mathrm{Um}$ estudo do grupo europeu de transplante de medula óssea e sangue periférico (EBMT) mostrou que a sobrevida após a recidiva citogenética ou hematológica estava associada a cinco fatores: tempo entre o diagnóstico e o transplante, fase da 
doença no transplante e na recidiva, tempo entre o transplante e a recidiva e tipo de doador. ${ }^{28}$ Os efeitos dos fatores de risco adversos individuais foram cumulativos. Desta maneira, os pacientes com dois ou mais fatores tiveram uma redução da sobrevida significativa ( $35 \%$ vs $65 \%$ em cinco anos). Além do mais, o DLI foi menos efetivo nos pacientes que desenvolveram DECH após o transplante. Entretanto, para os pacientes transplantados e recidivados em fase crônica, a eficácia da dose escalonada do DLI foi muito alta, atingindo mais de $90 \%$, com uma mortalidade relacionada ao procedimento de $5 \%$. Os autores consideraram o DLI como tratamento padrão no manejo das recidivas neste grupo de pacientes.

Simula et $a^{27}$ avaliaram a dose de células efetivas necessárias para atingir a remissão molecular e quais os fatores que influenciaram a dose efetiva nos pacientes que receberam o DLI, utilizando um esquema de dose escalonada. A proporção de pacientes que conseguiram a remissão molecular foi de $88 \%$. A proporção cumulativa dos respondedores aumentava significativamente após cada nível de dose. Utilizando uma dose de $\mathrm{CD}^{+} \geq 10^{7} / \mathrm{kg}, 56 \%$ dos pacientes em recidiva molecular/citogenética conseguiram remissão molecular, enquanto somente $20 \%$ daqueles em recidiva hematológica atingiram o mesmo resultado. Com uma mesma dose de células, $58 \%$ dos pacientes que receberam linfócitos de doadores não-aparentados conseguiram remissão molecular, comparados com $29 \%$ daqueles que receberam linfócitos de doadores irmãos. Os autores concluíram que a resposta ao DLI é dose dependente e que a dose de células efetivas é influenciada pela quantidade, pela fase da recidiva e pelo grau de histocompatibilidade entre doador e receptor.

O MI é agora uma alternativa ao DLI já que ele pode ser usado para a obtenção da remissão, sem a DECH, e pode ser efetivo quando o DLI falha. Ele também pode ser usado em combinação com doses baixas de DLI para aumentar a resposta, enquanto diminui os riscos da DECH.

Muitos grupos atualmente usam o MI para o tratamento de pacientes que recidivam após um TCTH. Muitos pacientes foram tratados em recidiva da doença em fase avançada, já que o DLI nesta situação tem um valor limitado. Outros foram tratados em recidiva citogenética ou hematológica, freqüentemente na presença de imunossupressão para o tratamento da DECH e/ou falência do DLI. ${ }^{29-}$ ${ }^{32} \mathrm{O}$ EBMT reportou uma análise retrospectiva de 128 pacientes tratados com MI para recidiva após um TCTH..$^{29} \mathrm{~A}$ resposta hematológica global foi de $84 \%$ (98\% para os pacientes em FC). A resposta citogenética completa foi de $58 \%$ para os pacientes em FC, $48 \%$ na fase avançada e $22 \%$ na CB. A resposta molecular completa (definida como ausência do transcrito $B C R-A B L$ ) foi obtida em 25 pacientes ( $26 \%$ ), dos quais 21 estavam em FC ou em fase mais avançada. Com uma mediana de acompanhamento de nove meses, a sobrevida em dois anos para os pacientes em FC, fase avan- çada e CB foi de $100 \%, 86 \%$ e $12 \%$, respectivamente. De 79 pacientes avaliados, $45(57 \%)$ atingiram quimerismo completo e 11 (14\%) quimerismo misto.

Weisser $e a^{l 32}$ compararam o uso de DLI ou MI em 31 pacientes. Vinte e um pacientes foram tratados com DLI (14 com recidiva citogenética e sete com recidiva hematológica) e dez com MI por falta de disponibilidade do doador original (nove com citogenética e um com hematológica). A resposta molecular completa foi observada em vinte dos 21 pacientes (95\%) que receberam DLI e em sete dos dez $(70 \%)$ que receberam MI. Entretanto, seis dos dez pacientes tratados com MI perderam a sua melhor resposta durante o tratamento. O MI foi descontinuado em quatro pacientes com resposta molecular completa e a doença recidivou em três pacientes num período de 3-4 meses após. Sete pacientes que foram tratados com o MI receberam subseqüentemente o DLI e seis conseguiram resposta molecular completa. Os autores concluíram que o MI, diferente do DLI, não induz respostas duradouras na maioria dos pacientes.

Savani et $a l^{13}$ testaram se a combinação do DLI e MI poderia ser mais efetiva em 37 pacientes com recidiva após um TCTH (10 com recidiva molecular, 14 hematológica e 13 em fase avançada). Os autores confirmaram que o MI é seguro e bem tolerado em combinação com o DLI. Além do mais, foi observado um efeito benéfico da associação do DLI e MI comparado com o uso isolado de cada agente. Com a combinação, os pacientes atingiram a resposta molecular completa mais rapidamente, foram capazes de parar o MI sem recidiva molecular e tiveram uma sobrevida livre de doença maior. A combinação também pareceu ser mais efetiva para a obtenção da resposta molecular completa, quando comparada ao tratamento único, naqueles pacientes com doença em fase mais avançada. Os autores concluíram que o DLI parece ter ação sinérgica com o MI para induzir uma resposta molecular rápida e duradoura.

\section{Recomendações}

- O MI pré-transplante parece não aumentar a toxicidade precoce ou atrasar a pega.

- O MI pré-transplante parece não piorar a sobrevida, recidiva e mortalidade não relacionada à recidiva.

- Entretanto, pacientes em FC com resposta citogenética subótima, ou perda da resposta citogenética, pré-transplante, têm sobrevida pior. Desta maneira, evitar tranplantar pacientes em FC com resposta subótima ou com perda da resposta citogenética.

- A resposta ao DLI é dependente da dose.

- A dose de célula efetiva é influenciada pela fase da LMC na recidiva e se o doador é aparentado ou não aparentado.

- Imatinibe é hoje uma alternativa ao DLI.

- Imatinibe pode ser útil quando não se tem resposta ao DLI, ou durante o tratamento da DECH. 
- A resposta ao MI é rápida e durável na recidiva em FC.

- A resposta é menos efetiva na recidiva em FA ou CB.

- O DLI associado ao MI parece induzir remissão molecular rápida e duradour.a

- Tratar a recidiva molecular com DLI.

- Tratar as recidivas citogenética, hematológica, fase acelerada ou crise blástica com MI associado ao DLI.

\section{Abstract}

Imatinib mesylate (IM) has become the first-line therapy for chronic myeloid leukemia (CML). Allogeneic hematopoietic stem cell transplantation (HSCT) is increasingly chosen as salvage therapy for patients who are intolerant of IM, fail to achieve a complete cytogenetic response (CCR) or relapse. The first part of this review will discuss the effect of prior exposure to IM on transplant outcomes and the impact of a poor or a loss of response at the time of transplantation on post-transplantation survival of patients who underwent transplantation in a chronic phase $(C P)$. The second part will discuss the management of relapse disease after transplant. Donor lymphocyte infusion (DLI) has become the treatment of choice for patients who relapse. The response to DLI is dosedependent and the effective cell dose is influenced by the quantity and phase of CML at relapse and degree of donor/recipient histocompatibility. IM is now an alternative to DLI as it can be used to achieve remission without graft-versus-host disease and may be effective when DLI has failed. It can also be used in combination with lower doses of DLI to maximize responses. IM is safe and well tolerated in combination with DLI, patients achieve molecular response more rapidly, are able to stop IM without recurrence of molecular disease and this treatment has a higher disease free survival rate after transplantation. Rev. bras. hematol. hemoter. 2008; 30(Supl.1):47-51.

Key words: Imatinib mesylate; chronic myeloid leukemia; allogeneic hematopoietic stem cell transplantation; donor lymphocyte infusion; relapse.

\section{Referências Bibliográficas}

1. Oehler VG, Gooley T, Snyder DS, et al. The effects of imatinib mesylate treatment before allogeneic transplantation for chronic myeloid leukemia. Blood. 2007;109:1782-9.

2. Hochhaus A, Druker BJ, Larson RA, et al. IRIS 6-year follow-up; Sustained survival and declining annual rate of transformation in patients with newly diagnosed chronic myeloid leukemia in chronic phase (CML-CP) treated with Imatinib. Blood. 2007; $110: 25 \mathrm{a}$.

3. Kantarjian HM, Cortes J, OBrien S, et al. Imatinib mesylate (STI571) therapy for Philadelphia chromosome-positive chronic myelogenous leukemia in blast phase. Blood. 2002;99:3547-53.

4. Kantarjian HM, Talpaz M, OBrien S, et al. Imatinib mesylate for Philadelphia chromosome-positive, chronic-phase myeloid leukemia after failure of interferon-alpha: follow-up results. Clin Cancer Res. 2002;8:2177-87.

5. Sawyers CL, Hochhaus A, Feldman E, et al. Imatinib induces hematologic and cytogenetic responses in patients with chronic myelogenous leukemia in myeloid blast crisis: results of a phase II study. Blood. 2002;99:3530-9.
6. Talpaz M, Silver RT, Druker BJ, et al. Imatinib induces durable hematologic and cytogenetic responses in patients with accelerated phase chronic myeloid leukemia: results of a phase 2 study. Blood. 2002;99:1928-37.

7. Cortes J, Giles F, OBrien S, et al. Result of high-dose imatinib mesylate in patients with Philadelphia chromosome-positive chronic myeloid leukemia after failure of interferon-alpha. Blood. 2003;102:83-6.

8. Radich JP, Gooley T, Bensinger W, et al. HLA-matched related hematopoietic cell transplantation for chronic-phase CML using a targeted busulfan and cyclophosphamide preparative regimen. Blood. 2003;102:31-5.

9. Hansen JA, Gooley TA, Martin PJ, et al. Bone marrow transplants from unrelated donors for patients with chronic myeloid leukemia. N Engl J Med. 1998;338:962-8.

10. Barrett J. Allogeneic stem cell transplantation for chronic myeloid leukemia. Semin Hematol. 2003;40:59-71.

11. Clift RA, Buckner CD, Thomas ED, et al. Marrow transplantation for patients in accelerated phase of chronic myeloid leukemia. Blood. 1994;84:4368-73.

12. Hughes TP, Kaeda J, Branford S, et al. Frequency of major molecular responses to imatinib or interferon alfa plus cytarabine in newly diagnosed chronic myeloid leukemia. N Engl J Med. 2003;349: 1423-32.

13. Radich JP, Gehly G, Gooley T, et al. Polymerase chain reaction detection of the BCR-ABL fusion transcript after allogeneic marrow transplantation for chronic myeloid leukemia: results and implications in 346 patients. Blood. 1995;85:2632-8.

14. Radich JP, Gooley T, Bryant E, et al. The significance of bcr-abl molecular detection in chronic myeloid leukemia patients "late," 18 months or more after transplantation. Blood. 2001;98:1701-7.

15. Zander AR, Zabelina T, Renges $H$, et al. Pretreament with glivec increases transplant-related mortality after allogeneic transplant. Blood 2003;102:468a.

16. Shimoni A, Kroger N, Zander AR, et al. Imatinib mesylate (STI571) in preparation for allogeneic hematopoietic stem cell transplantation and donor lymphocyte infusions in patients with Philadelphia-positive acute leukemias. Leukemia. 2003; 17:290-7.

17. Zaucha JM, Prejzner W, Giebel S, et al. Imatinib therapy prior to myeloablative allogeneic stem cell transplantation. Bone Marrow Transplant. 2005;36:417-24.

18. Deininger M, Schleuning M, Greinix H, et al. The effect of prior exposure to imatinib on transplant-related mortality. Haematologica. 2006;91:452-9.

19. Wassmann B, Pfeifer H, Scheuring U, et al. Therapy with imatinib mesylate (Glivec) preceding allogeneic stem cell transplantation (SCT) in relapsed or refractory Philadelphia-positive acute lymphoblastic leukemia (Ph+ALL). Leukemia. 2002;16:2358-65.

20. Kim DW, Chung YJ, Lee S, et al. Pretransplant imatinib can improve the outcome of nonmyeloablative stem cell transplantation without increasing the morbidity in Philadelphia chromosome-positive chronic myeloid leukemia. Leukemia. 2004;18:1907-9.

21. Bornhauser M, Kroger N, Schwerdtfeger R, et al. Allogeneic haematopoietic cell transplantation for chronic myelogenous leukaemia in the era of imatinib: a retrospective multicentre study. Eur J Haematol. 2006;76:9-17.

22. Seggewiss R, Lore K, Greiner E, et al. Imatinib inhibits T-cell receptor-mediated T-cell proliferation and activation in a dosedependent manner. Blood. 2005;105:2473-9.

23. Appel S, Balabanov S, Brummendorf TH, Brossart P. Effects of imatinib on normal hematopoiesis and immune activation. Stem Cells. 2005;23:1082-8. 
24. Kolb HJ, Mittermuller J, Clemm C, et al. Donor leukocyte transfusions for treatment of recurrent chronic myelogenous leukemia in marrow transplant patients. Blood. 1990; 76: 2462-5.

25. Guglielmi C, Arcese W, Dazzi F, et al. Donor lymphocyte infusion for relapsed chronic myelogenous leukemia: prognostic relevance of the initial cell dose. Blood. 2002;100:397-405.

26. Gilleece MH, Dazzi F. Donor lymphocyte infusions for patients who relapse after allogeneic stem cell transplantation for chronic myeloid leukaemia. Leuk Lymphoma. 2003;44:23-8.

27. Simula MP, Marktel S, Fozza C, et al. Response to donor lymphocyte infusions for chronic myeloid leukemia is dose-dependent: the importance of escalating the cell dose to maximize therapeutic efficacy. Leukemia. 2007;21:943-8.

28. Guglielmi C, Arcese W, Hermans J, et al. Risk assessment in patients with $\mathrm{Ph}+$ chronic myelogenous leukemia at first relapse after allogeneic stem cell transplant: an EBMT retrospective analysis. The Chronic Leukemia Working Party of the European Group for Blood and Marrow Transplantation. Blood. 2000;95:3328-34.

29. Olavarria E, Ottmann OG, Deininger M, et al. Response to imatinib in patients who relapse after allogeneic stem cell transplantation for chronic myeloid leukemia. Leukemia. 2003;17:1707-12.

30. DeAngelo DJ, Hochberg EP, Alyea EP, et al. Extended follow-up of patients treated with imatinib mesylate (gleevec) for chronic myelogenous leukemia relapse after allogeneic transplantation: durable cytogenetic remission and conversion to complete donor chimerism without graft-versus-host disease. Clin Cancer Res. 2004;10:5065-71.

31. Savani BN, Montero A, Kurlander R, Childs R, Hensel N, Barrett AJ. Imatinib synergizes with donor lymphocyte infusions to achieve rapid molecular remission of CML relapsing after allogeneic stem cell transplantation. Bone Marrow Transplant. 2005;36:1009-15.

32. Weisser M, Tischer J, Schnittger S, Schoch C, Ledderose G, Kolb HJ. A comparison of donor lymphocyte infusions or imatinib mesylate for patients with chronic myelogenous leukemia who have relapsed after allogeneic stem cell transplantation. Haematologica. 2006;91:663-6.

O tema apresentado e o convite ao autor constam da pauta elaborada pelos co-editores, Professor Ricardo Pasquini e

Professor Cármino Antonio de Souza.

Avaliação: Co-editores e um revisor externo.

Publicado após revisão e concordância do editor.

Conflito de interesse: não declarado.

Recebido: 21/02/2008

Aceito: $22 / 02 / 2008$ 